\title{
Influence of lingual bracket position on microbial and periodontal parameters in vivo
}

\author{
Maria Francesca SFONDRINI ${ }^{1}$, Maurizia DEBIAGGI ${ }^{2}$, Francesca ZARA ${ }^{2}$, Roberto BRERRA ${ }^{2}$, Mario COMELLI $^{3}$, Marco \\ BIANCHI ${ }^{4}$, Sara Ramella POLLONE ${ }^{5}$, Andrea SCRIBANTE ${ }^{6}$
}

1- MD, DDS, PhD, Professor, Department of Orthodontics, University of Pavia, Pavia, Italy.
2- MD, Professor, Department of Microbiology, University of Pavia, Pavia, Italy.
3- MD, Professor, Department of Statistics, University of Pavia, Pavia, Italy.
4- DDS, Graduate Fellow, Department of Orthodontics, University of Pavia, Pavia, Italy.
5- DDS, Graduate Fellow, Department of Orthodontics, University of Pavia, Pavia, Italy.
6- DDS, PhD, Research Resident, Department of Orthodontics and Department of Surgical Sciences, University of Pavia, Pavia, Italy.

Corresponding address: Dr. Andrea Scribante - Istituto di Discipline Odontostomatologiche "S. Palazzi" - IRCCS Policlinico S. Matteo - P.le Golgi 2 - 27100 Pavia - Italy - Phone/fax: +39-0382-516223 - e-mail: andrea.scribante@unipv.it

Received: October 18, 2010 - Modification: August 4, 2011 - Accepted: August 16, 2011

\section{ABSTRACT}

\begin{abstract}
$\mathrm{O}$ bjective: Lingual orthodontics is becoming more popular in dental practice. The purpose of the present investigation was to compare plaque formation on teeth bonded with the same bracket onto buccal or lingual surface, with non-bonded control teeth, via an in vivo growth experiment over a 30-day period. Material and Methods: A randomized controlled trial with split-mouth design was set up enrolling 20 dental students. Within each subject sites with buccal and lingual brackets and control sites were followed. Clinical periodontal parameters (periodontal pocket depth: PPD; bleeding on probing: BOP) were recorded at baseline and on days 1, 7 and 30. Microbiological samples were taken from the brackets and the teeth on days 1,7 and 30 to detect colony-forming units (CFU). Total CFU, streptococci CFU and anaerobe CFU were measured. Results: No significant differences $(P>0.05)$ were found between buccal and lingual brackets in terms of clinical periodontal parameters and microbiological values. Conclusion: Bracket position does not have significant impact on bacterial load and on periodontal parameters.
\end{abstract}

Key words: Microbiology. Orthodontics. Dental plaque. Periodontal diseases.

\section{INTRODUCTION}

Gingivitis and periodontal disease are frequent concomitant phenomena of orthodontic treatment with fixed appliances ${ }^{29}$. It seems that the main factor for an increased accumulation of dental plaque and inflammatory response is the appearance of new retentive places around the components of fixed appliances attached to the teeth ${ }^{1}$. Several studies have addressed the impact of fixed, removable, and myofunctional orthodontic/orthopedic appliances or retainers in relation to supragingival plaque accumulation and gingivitis ${ }^{3,11,12}$.

The quantity, as well as the quality of plaque, is influenced by many factors including surface characteristics ${ }^{23,24}$, surface roughness and surface free energy ${ }^{21}$ and bracket design ${ }^{33}$, frequency of sucrose exposition ${ }^{25}$. The presence of gingival inflammation will further increase plaque growth ${ }^{22,26}$.

Because of its outstanding aesthetic preconditions and its growing practicability, lingual orthodontics accounts for an ever-increasing percentage of orthodontic treatments ${ }^{20,28}$. Oral hygiene is even more important for therapy with lingual brackets than for therapy with labial brackets because control is more difficult from the lingual face than from the buccal face, and plaque accumulations, gingivitis, and demineralization are not detected by the patient ${ }^{14}$. Most microbiological investigations have been performed during orthodontic treatment ${ }^{4,9,15,33}$ but there are no studies dealing with the difference between buccal and lingual brackets.

Accordingly, the purpose of the present investigation was to evaluate the influence of buccal and lingual orthodontic appliances on microbiological and periodontal parameters of bonded teeth. The null 
Table 1- Study population with data on gender distribution, previous orthodontic treatment and age

\begin{tabular}{cccccc}
\hline & N & $\begin{array}{c}\text { Previous treatment with } \\
\text { fixed appliances }\end{array}$ & $\begin{array}{c}\text { No previous treatment } \\
\text { with fixed appliances }\end{array}$ & Age (mean) & Age (SD) \\
\hline Male & 6 & 3 & 3 & 23.3 & 2.5 \\
Female & 14 & 10 & 4 & 24.2 & 2.5 \\
Total & 20 & 13 & 7 & 23.8 & 2.4 \\
\hline
\end{tabular}

$\mathrm{SD}=$ standard deviation

\begin{tabular}{|l|l|l|l|l|l|l|l|l|l|l|}
\hline B & & & & B & & L & & & & L \\
\hline & L & & L & & & & B & & B & \\
\hline
\end{tabular}

Figure 1- The four different clinical conditions of the splitmouth design

hypothesis of the study was that there is no significant difference in terms of microbiological environment and clinical periodontal parameters between buccal and lingual brackets.

\section{MATERIAL AND METHODS}

\section{Subjects}

Twenty dental students ( 14 females and 6 males, Caucasians aged between 20 and 32 years) were involved in the study (Table 1 ). They were given a written explanation of the background of the study and its objectives. After screening for their suitability and after good comprehension of the protocol, they all gave their written informed consent. During the experiment, the participants could always contact the researcher for questions or remarks. Before the study, all students received oral hygiene instructions in order to ensure a healthy periodontal situation.

The initial placement of the brackets was performed via a randomized protocol by means of concealed envelopes. The students were selected to fulfill the following inclusion criteria: no smoking, absence of extensive dental restorations or adhesive-fixed partial dentures, a sulcus bleeding index ${ }^{18}$ of $<0.3$ and no antibiotics during or up to 4 months before the study. The students were also asked whether they had already received an orthodontic treatment with fixed appliances because this might have consequences for smoothness of the buccal enamel ${ }^{10}$ and as such on the microbial adhesion in the early formation of a dental plaque film ${ }^{23,24}$. The Ethics Committee approved the design of this study.

\section{Experimental procedure \\ Experimental design}

The study had a randomized, examiner-blind, split-mouth design ${ }^{33}$. In every student, the mouth was divided into four quadrants, two of which served as controls. One type of bracket (2D, Forestadent, Pforzheim, Germany) was bonded in two different sites: buccal and lingual. For the split-mouth comparison, 8 sites were defined, namely the canine and the first premolar of each quadrant. The brackets were placed in contralateral antagonistic quadrants. The first quadrant used for bracket placement and the order in which the brackets were placed were randomly chosen by means of concealed envelopes, the second one was at the other side of the mouth in the antagonistic jaw. The buccal and lingual bonded teeth were alternated, giving rise to four different experimental settings (Figure 1 ):

-buccal position in the first quadrant, lingual position in the third quadrant;

-buccal position in the second quadrant, lingual position in the fourth quadrant;

-buccal position in the third quadrant, lingual position in the first quadrant;

-buccal position in the fourth quadrant, lingual position in the second quadrant.

The teeth bonded with the different brackets were compared with each other and with the non-bonded control sites.

During the study period, the students visited the clinic three times: T0 (baseline): to record the status of the periodontium (periodontal pocket depth: PPD; bleeding on probing: BOP), to collect samples of dental plaque from the teeth and to place the brackets; T1 (day 7): to record the periodontal status (PPD and BOP) and to collect from the test teeth and from control teeth; T2 (day 30): to record the periodontal status (PPD and BOP), to collect samples of dental plaque from the test teeth and the control teeth, and to remove the brackets.

\section{Bracket placement/removal}

The teeth were rinsed, dried with an oil-free air syringe, and etched with $37 \%$ phosphoric acid for 30 s. After a thorough washing, they were completely dried with an oil-free air syringe. Then, stainless steel brackets (2D, Forestadent, Pforzheim, Germany) were bonded to the selected teeth with an adhesive system (Transbond XT, 3M, Monrovia, CA, USA), according to the manufacturer's directions. After applying the primer on the etched enamel, a small amount of composite resin was placed on the mesh pad of the bracket. The bracket was positioned on the buccal or lingual surface of the teeth with sufficient pressure to squeeze excess adhesive, which was 
removed from the margins of the bracket base with an explorer before polymerization. Bracket was then light-cured with a visible light-curing unit (Ortholux XT, 3M Unitek) for $10 \mathrm{~s}$ on the mesial side of the bracket and for $10 \mathrm{~s}$ on the distal side (total cure time $20 \mathrm{~s}$ ). All brackets were bonded by the same operator. Verbal and written instructions regarding the appliance care and hygiene protocols were issued to each patient, along with a specific request to return if a bracket became loose or if any problem arose with the appliance.

\section{Periodontal parameters}

PPD and BOP were scored at baseline, day 7 and day 30. To record the PPD, a millimeter probe (HUfriedly Pc puns, Chicago, IL, USA) was inserted in the gingival sulcus. The pocket depths were measured at the buccal, lingual, mesial and distal sides of the tooth and rounded off to the nearest $0.5 \mathrm{~mm}$. BOP was recorded (0: absent; 1: present) $24 \mathrm{~s}$ after determination of PPD. The examiner was blinded to previous scores.

\section{Culture techniques}

At baseline, on days seven and thirty microbial samples were also taken using a sterile curette (SG $5 / 6 \mathrm{HU}$-Friedy) from the test and the control teeth. The supra-gingival dental plaque was taken away with sterile curettes. This was carried out without traumatizing the gingiva and without disturbing the plaque film on the remaining sites ${ }^{33}$. The supragingival plaque samples were transferred into flip-capped vials containing $250 \mu \mathrm{L}$ of reduced transport fluid (RTF) ${ }^{30}$. All samples were transferred to the laboratory and processed within $3 \mathrm{~h}$.

The samples were pooled in $250 \mu \mathrm{L}$ of RTF and serial ten-fold dilutions were prepared in the same medium. Dilutions of $10^{-3}-10^{-5}$ were plated in duplicate using a spiral plater onto three different media: Mitis-salivarius (MS) agar was used to determine the total count of streptococci, mitis-salivarius-bacitracin (MSB) agar was used as the selective medium for differentiation and enumeration quantification of $S$. mutans and CDC Anaerobe 5\% sheep blood agar (BD) for enumeration of total recoverable anaerobic bacteria.

After 3 days of aerobic incubation at $37^{\circ} \mathrm{C}$ for MS and MSB agar plates and 6 days of anaerobic incubation (Gas Pack EZ system, BD) at $37^{\circ} \mathrm{C}$ for blood agar plates, the number of colony-forming units (CFU) was counted. The total count of microorganism was determined on countable (from 30 to 300 colonies) plates.

\section{Statistical analysis}

The $P$ values report concern the interaction between time and position of the bracket, included in mixed linear models fitted to the microbiological and clinical outcomes. The models included other possibly relevant fixed effects (e.g. side of the mouth), and the patient identity as a random effect. The $\mathrm{R}$ statistical package (R Development Core Team, Wien, Austria) was used for computation. The significance level was set at $5 \%$.

\section{RESULTS}

\section{Microbiological parameters}

The numbers of streptococci, anaerobic and total CFU in supragingival plaque samples during the experimental period showed no significant differences days combined (Table 2). Buccal sites in general allowed equal plaque formation than the lingual sites $(P>0.05)$.

In Table 3, the results are separately depicted per day and per material. Buccal sites showed no significant differences from lingual sites $(P>0.05)$ for either streptococci, anaerobe or total CFU counts.

Table 2- Amount of colony-forming units (CFU) per site, days combined. The upper part displays the means per site; the lower part the differences between the sites with the corresponding P-values. SD= standard deviation

\begin{tabular}{|c|c|c|c|c|c|c|}
\hline & $\begin{array}{l}\text { CFU Total } \\
\text { (mean) }\end{array}$ & $\begin{array}{l}\text { CFU Total } \\
\text { (SD) }\end{array}$ & $\begin{array}{l}\text { Streptococci } \\
\text { CFU (mean) }\end{array}$ & $\begin{array}{l}\text { Streptococci } \\
\text { CFU (SD) }\end{array}$ & $\begin{array}{c}\text { Anaerobe CFU } \\
\text { (mean) }\end{array}$ & $\begin{array}{c}\text { Anaerobe CFU } \\
\text { (SD) }\end{array}$ \\
\hline \multicolumn{7}{|c|}{ Means per site } \\
\hline V sites & $5.21 \mathrm{E}+6$ & $1.61 \mathrm{E}+7$ & $6.36 \mathrm{E}+3$ & $1.20 \mathrm{E}+4$ & $1.11 \mathrm{E}+7$ & $2.93 \mathrm{E}+7$ \\
\hline L sites & $9.86 \mathrm{E}+6$ & $2.74 \mathrm{E}+7$ & $1.75 \mathrm{E}+5$ & $7.62 E+5$ & $9.61 \mathrm{E}+6$ & $2.66 \mathrm{E}+7$ \\
\hline \multirow[t]{2}{*}{ Control sites } & $5.63 \mathrm{E}+7$ & $2.55 \mathrm{E}+8$ & $1.12 \mathrm{E}+6$ & $6.36 \mathrm{E}+6$ & $1.14 \mathrm{E}+7$ & $2.71 \mathrm{E}+7$ \\
\hline & Total CFU & $P$ value & $\begin{array}{l}\text { Streptococci } \\
\text { CFU }\end{array}$ & $P$ value & Anaerobe CFU & $P$ value \\
\hline \multicolumn{7}{|c|}{ Differences between sites } \\
\hline V-L & $4.65 \mathrm{E}+6$ & 0.68 & $1.69 \mathrm{E}+5$ & 0.43 & $-1.49 E+6$ & 0.3 \\
\hline V-Control & $5.11 \mathrm{E}+7$ & 0.2 & $1.11 \mathrm{E}+6$ & 0.96 & $3.00 \mathrm{E}+5$ & 0.07 \\
\hline L-Control & $4.64 \mathrm{E}+7$ & 0.41 & $9.45 E+5$ & 0.38 & $1.79 E+6$ & 0.51 \\
\hline
\end{tabular}


Table 3- The mean pocket depth (PPD) measurements in millimeters, displayed per day and per site with the corresponding standard deviations (SD)

\begin{tabular}{cccc}
\hline Day & $\begin{array}{c}\text { Bracket } \\
\text { position }\end{array}$ & $\begin{array}{c}\text { PPD } \\
\text { (mean) }\end{array}$ & $\begin{array}{c}\text { PPD } \\
\text { (SD) }\end{array}$ \\
\hline 1 & B sites & 1.2 & 0.62 \\
& L sites & 1.22 & 0.49 \\
& Control sites & 1.18 & 0.53 \\
\hline & B sites & 1.35 & 0.46 \\
\hline 30 & L sites & 1.24 & 0.49 \\
& Control sites & 1.19 & 0.42 \\
& B sites & 1.38 & 0.41 \\
& L sites & 1.43 & 0.45 \\
& Control sites & 1.32 & 0.42 \\
\hline
\end{tabular}

Periodontal parameters

PPD

No significant inter-material differences in PPD were present among the various groups. No significant increase in PPD was recorded $(P>0.05)$ on days 7 and 30 for either buccal or lingual bracket position and for control sites.

\section{BOP}

Only few BOP sites were recorded during the experimental period. No significant differences $(P>0.05)$ in BOP sites were detected among the different groups at different times.

No adverse events were reported.

\section{DISCUSSION}

The null hypothesis of the study was partially rejected. The present experiment with split-mouth design did not detect any significant difference in periodontal and microbiological parameters between the bonded teeth and the non-bonded control teeth or between the groups with brackets bonded onto buccal and lingual sides.

A number of studies have investigated the influence of orthodontic therapy and appliances on the oral microbial flora. These changes could potentially have a significant impact on patient oral health, including gingival inflammation and demineralization of teeth ${ }^{2}$. Moreover, in literature, orthodontic therapy was associated with 0.03 millimeters of gingival recession, $0.13 \mathrm{~mm}$ of alveolar bone loss and 0.23 $\mathrm{mm}$ of increased pocket depth when compared with no treatment ${ }^{6}$. The effects of orthodontic therapy on gingivitis and attachment loss are inconsistent across studies ${ }^{6}$.

Anhoury, et al. ${ }^{2}$ (2002) evaluated microbial profile on metallic and ceramic bracket materials and found that composition of dental plaque formed on each bracket type is very similar between the two bracket types and may be of limited clinical significance. Furthermore, the differences detected do not favor one bracket type over another with respect to bacterial accumulation.

Other authors ${ }^{33}$ evaluated influence of bracket design on microbiological and periodontal parameters showing both anaerobe and aerobe colony-forming units significantly higher for self-ligating brackets than for conventional brackets. No significant differences for bleeding on probing were observed.

Microbial and periodontal parameters have been evaluated also for orthodontic bands ${ }^{7}$, conventional stainless steel brackets ${ }^{19}$, ceramic attachments ${ }^{2}$, and self-ligating brackets. In literature there are no studies about lingual brackets. These brackets have been investigated about their laboratory and clinical processes and for particular recommendations on oral hygiene ${ }^{14,20,28}$. Those authors showed that special emphasis should be placed on the oral hygiene of patients with lingual brackets and that excellent oral hygiene is possible in patients with lingual devices after instruction and motivation ${ }^{14}$.

The results of the present investigation showed no significant differences in total CFU, streptococci CFU and anaerobe CFU counts among buccal, lingual and control sites. This is in agreement with a previous investigation that evaluated CFU of buccal brackets versus control non-bonded sites and showed no significant differences between the two groups ${ }^{32}$. There are also authors that found significant CFU count decrease ${ }^{29}$ or increase $7,16,19,27$ in bracket sites compared with control sites. This variability is probably due to the different testing conditions and multiple variables correlated with clinical researches.

Moreover, in the present study, PPD and BOP measurements were analyzed and showed no significant differences among buccal, lingual and control sites. This is in agreement with previous studies that evaluated these periodontal parameters using conventional brackets: all of them showed no significant increase of $\mathrm{BOP}^{12,14}$ and $\mathrm{PPD}^{12,19,33}$ after brackets placement. There are also studies that detected significant increase of $\mathrm{PPD}^{27}$ and BOP19,33 after bracket placement. This variability could be due to different orthodontic bracket materials that have dissimilar clinical manifestations.

The limits of the present study would be that the population of the present investigation was represented by dental students, who are expected to maintain better oral hygiene than the general population. Hygiene regimen of subjects in the trial can be reached also in the general population only if adequately motivated ${ }^{8}$. In fact, even if in recent decades, decreasing prevalence in dental caries has been observed worldwide ${ }^{5}$ correct teaching of hygiene protocols is crucial, especially during orthodontic treatment. Therefore, even if in the present investigation buccal or lingual bracket position did not have significant impact on PPD and BOP periodontal parameters, dental health promotion should be fully 
integrated into broadly based health-promoting strategies. Oral hygiene remain the major factor when evaluating caries prevention and dental biofilm ${ }^{13,17,31}$.

Another issue is represented by the presence of wires used during the regular orthodontic treatment. The wires and brackets work together to limit hygiene and therefore a further development of the present study could include also this aspect.

Future investigations should be performed to visualize the potentially different periodontal parameters correlated with different orthodontic bracket systems so that brackets can be designed to reduce plaque adhesion.

\section{CONCLUSIONS}

This study demonstrated the following: 1 . buccal or lingual bracket position does not have a significant impact on PPD and BOP periodontal parameters; buccal or lingual bracket position does not have significant impact on streptococci, anaerobe and total CFU counts.

\section{ACKNOWLEDGEMENTS}

The authors wish to thank $3 \mathrm{M}$ and Forestadent for providing the materials tested in this study.

\section{REFERENCES}

1- Alexander SA. Effects of orthodontic attachments on the gingival health of permanent second molars. Am J Orthod Dentofacial Orthop. 1991;100:337-40.

2- Anhoury P, Nathanson D, Hughes CV, Socransky S, Feres M, Chou LL. Microbial profile on metallic and ceramic bracket materials. Angle Orthod. 2002;72:338-43.

3- Artun J, Urbye KS. The effect of orthodontic treatment on periodontal bone support in patients with advanced loss of marginal periodontium. Am J Orth Dentofacial Orthop. 1998;93:143-8.

4- Atack NE, Sandy JR, Addy M. Periodontal and microbiological changes associated with the placement of orthodontic appliances. A review. J Periodontol. 1996;67:78-85.

5- Baldini V, Tagliaferro EP, Ambrosano GM, Meneghim MD, Pereira AC. Use of occlusal sealant in a community program and caries incidence in high- and low-risk children. J Appl Oral Sci. 2011;19(4):396-402. 6- Bollen AM, Cunha-Cruz J, Bakko DW, Huang GJ, Hujoel PP. The effects of orthodontic therapy on periodontal health: a systematic review of controlled evidence. J Am Dent Assoc. 2008;139:413-22. 7- Boyd R, Baumrind S. Periodontal consideration in the use of bonds or bands on molar in adolescents and adults. Angle Orthod. $1992 ; 62 ; 117-26$.

8- Carvalho VF, Okuda OS, Bernardo CC, Pannuti CM, Georgetti MA, De Micheli G, et al. Compliance improvement in periodontal maintenance. J Appl Oral Sci. 2010;18(3):215-9.

9- Diamanti-Kipioti A, Gusberti FA, Lang NP. Clinical and microbiological effects of fixed orthodontic appliances. J Clin Periodontol. 1987;14:326-33.

10- Eliades T, Gioka C, Eliades G, Makou M. Enamel surface roughness following debonding using two resin grinding methods. Eur J Orthod. 2004;26:333-8.

11- Glans R, Larsson E, Øgaard B. Longitudinal changes in gingival condition in crowded and noncrowded dentitions subjected to fixed orthodontic treatment. Am J Orth Dentofacial Orthop. 2003;124:67982.
12- Gomes SC, Varela CC, Veiga SL, Rösing CK, Oppermann RV. Periodontal conditions in subjects following orthodontic therapy. A preliminary study. Eur J Orthod. 2007;29:477-81.

13- Gontijo L, Cruz RA, Brandão PR. Dental enamel around fixed orthodontic appliances after fluoride varnish application. Braz Dent J. 2007; 18:49-53.

14- Hohoff A, Stamm T, Kühne N, Wiechmann D, Haufe S, Lippold $\mathrm{C}$, et al. Effects of a mechanical interdental cleaning device on oral hygiene in patients with lingual brackets. Angle Orthod. 2003;73:57987.

15- Huser MC, Baehni PC, Lang R. Effects of orthodontic bands on microbiologic and clinical parameters. Am J Orthod Dentofacial Orthop. 1990;97:213-8.

16- Lee SM, Yoo SY, Kim HS, Kim KW, Yoon YJ, Lim SH, et al. Prevalence of putative periodontopathogens in subgingival dental plaques from gingivitis lesions in Korean orthodontic patients. J Microbiol. 2005;43:260-5.

17- Maltz M, Jardim JJ, Alves LS. Health promotion and dental caries. Braz Oral Res. 2010;24(Suppl 1):18-25.

18- Mühlemann HR, Son S. Gingival sulcus bleeding - a leading symptom in initial gingivitis. Helv Odontol Acta. 1971;15,107-13.

19- Naranjo AA, Triviño ML, Jaramillo A, Betancourth M, Botero JE. Changes in the subgingival microbiota and periodontal parameters before and 3 months after bracket placement. Am J Orthod Dentofacial Orthop. 2006;130:275.e17-22.

20- Poon KC, Taverne AA. Lingual orthodontics: a review of its history. Aust Orthod J. 1997;15:101-4.

21- Quirynen M, Bollen CM. The influence of surface roughness and surface free energy on supra- and subgingival plaque formation in man. A review of the literature. J Clin Periodontol. 1995;22:1-14.

22- Quirynen M, Dekeyser C, Van Steenberghe D. The influence of gingival inflammation, tooth type, and timing on the rate of plaque formation. J Periodontol. 1991;62:219-22.

23- Quirynen M, Marechal M, Busscher HJ, Weerkamp AH, Arends J, Darius $\mathrm{PL}$, et al. The influence of surface free-energy on planimetric plaque growth in man. J Dent Res. 1989;68:796-9.

24- Quirynen M, Marechal M, Busscher HJ, Weerkamp AH, Darius PL, Van Steenberghe $D$. The influence of surface free energy and surface roughness on early plaque formation. An in vivo study in man. J Clin Periodontol. 1990;17:138-44.

25- Raggio DP, Braga MM, Rodrigues JA, Freitas PM, Imparato JC, Mendes FM. Reliability and discriminatory power of methods for dental plaque quantification. J Appl Oral Sci. 2010;18:186-93.

26- Ramberg $P$, Axelsson $P$, Lindhe J. Plaque formation at healthy and inflamed gingival sites in young individuals. J Clin Periodontol. $1995 ; 22: 85-8$.

27- Ristic M, Vlahovic Svabic M, Sasic M, Zelic O. Effects of fixed orthodontic appliances on subgingival microflora. Int J Dent Hyg. 2008;6:129-36.

28- Rummel V, Wiechmann D, Sachdeva RCL. Precision finishing in lingual orthodontics. J Clin Orthod. 1999;13:101-13.

29- Speer C, Pelz K, Hopfenmuller W, Holtgrave EA Investigations on the influencing of the subgingival microflora in chronic periodontitis, a study in adult patients during fixed appliances therapy. J Orofac Orthop. 2004;65,34-47.

30- Syed SA, Loesche WJ. Survival of human dental plaque flora in various transport media. Appl Microbiol. 1972;24:638-44.

31- Teles RP, Teles FR. Antimicrobial agents used in the control of periodontal biofilms: effective adjuncts to mechanical plaque control? Braz Oral Res. 2009;23(Suppl 1):39-48.

32- Thornberg MJ, Riolo CS, Bayirli B, Riolo ML, Van Tubergen EA, Kulbersh R. Periodontal pathogen levels in adolescents before, during, and after fixed orthodontic appliance therapy. Am J Orthod Dentofacial Orthop. 2009;135:95-8.

33- Van Gastel J, Qurynen M, Teughels W, Coucke W, Carels C. Influence of bracket design on microbial and periodontal parameters in vivo. J Clin Periodontol. 2007;34,423-31. 